\title{
Uncertainty-Aware Sensor Deployment Algorithms for Surveillance Applications
}

\author{
Yi Zou and Krishnendu Chakrabarty \\ Department of Electrical andComputer Engineering \\ Duke University, Durham, NC 27708, USA
}

\begin{abstract}
We consider the sensor deployment problem in the context of uncertainty in sensor locations subsequent to airdropping. Sensor deployment in such scenarios is inherently non-deterministic and there is a certain degree of randomness associated with the location of a sensor in the sensor field. We present two algorithms for the efficient placement of sensors in a sensor field when the exact locations of the sensors are not known. These algorithms are targeted at average coverage as well as at maximizing the coverage of the most vulnerable regions in the sensor field. Experimental results for an example sensor field demonstrate the application of our approach.
\end{abstract}

\section{INTRODUCTION}

Wireless sensor networks that are capable of observing the environment, processing the data, and making decisions based on these observations, have recently attracted considerable attention [1]. Such networks can be used to monitor the environment, detect, classify and locate specific events, and track targets over a specific region. The topology of the sensor field, i.e., the locations of the sensors, determines to a large extent the quality of the coverage provided by the sensor network. However, even if the sensor locations are precomputed for optimal coverage and resource utilization, there are inherent uncertainties in the sensor locations when the sensors are dispersed, scattered, or airdropped. Thus a key challenge in sensor deployment is to determine an uncertaintyaware sensor field architecture that reduces cost and provides high coverage, even though the exact location of the sensors may not be controllable.

In applications such as battlefield surveillance and environmental monitoring, sensors may be dropped from airplanes. Such sensors cannot be expected to fall exactly at predetermined locations; rather there are regions where there is a high probability of a sensor being actually located (Fig. 1). In underwater deployment, sensors may move due to drift or water currents. Thus the position of sensors may not be exactly known and for every point in the sensor field, there is only a certain probability of a sensor being located at that point.

In this work, we present two algorithms for sensor deployment wherein we assumed that sensor positions are not exactly predetermined. We assume that the sensor locations are calculated before deployment and an attempt is made during the airdrop to place sensors at these locations; however, the sensor placement calculations and coverage optimization are based on a Gaussian model, which assumes that if a sensor is intended for a specific point $P$ in the sensor field, its exact location can be anywhere in a "cloud" surrounding $P$.

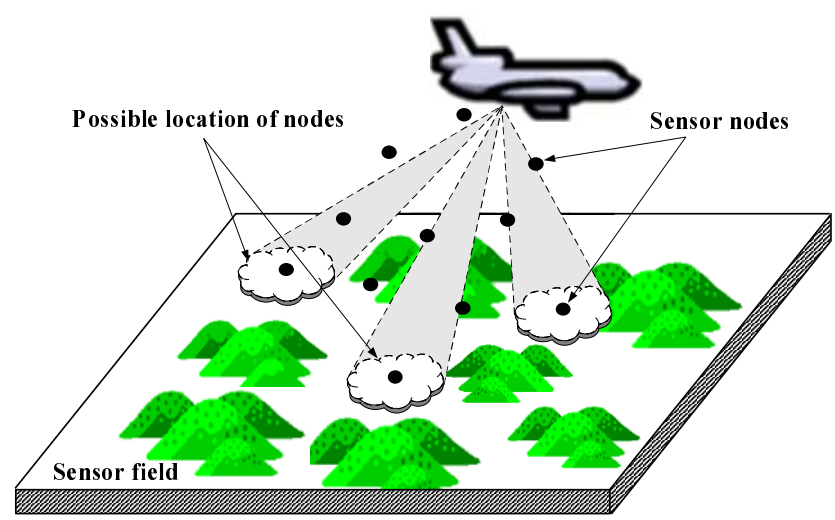

Fig. 1. Sensors dropped from airplanes. The clouded region gives the possible region of a sensor location. The black circle shows the mean (intended) position of a sensor.

We represent the sensor field as a grid (two- or threedimensional) of points. A target in the sensor field is therefore a logical object, which is represented by a set of sensors that see it. An irregular sensor field is modeled as a collection of grids. The optimization framework is however inherently probabilistic due to the uncertainty associated with sensor detections. We propose two algorithms for sensor placement that address coverage optimization under the constraints of imprecise detections and terrain properties. Note that the placement algorithms give us the sensor positions prior to actual placement and we assume that sensors are deployed in a single step. We limit our discussion in this paper to fixed sensors.

The remainder of the paper is organized as follows. In Section 2, we discuss some previous work in the field of sensor deployment. In Section 3, we describe our sensor detection model as well our approach for modeling the terrain. In Section 4, we describe the Gaussian model for sensor placement. Section 5 describes two procedures for placing sensors to provide adequate coverage. Section 6 presents experimental results. Finally, Section 7 concludes the paper and describes directions for future work.

\section{RELATED PRIOR WORK}

Related recent work has focused on the random deployment of sensors for target detection [2]. This method uses sequential deployment of sensors i.e., a limited number of sensors are deployed in each step until the desired minimum exposure 
or probability of detection of a target is achieved. In most practical applications however, we need to deploy the sensors in advance without any prior knowledge of the target and sequential deployment is often infeasible. Moreover, sequential deployment may be undesirable when the number of sensors or the area of the sensor field is large. Thus a single-step deployment scheme is more advantageous in such scenarios.

Related work on terrain model acquisition for motion planning has focused on the movement of a robot in an unexplored "sensor field" [3]. While knowledge of the terrain is vital for surveillance, it does not directly solve the sensor placement problem. The problem of determining the coverage provided by a given placement of sensors has also been discussed in the literature [5], [6]. An optimal polynomial-time algorithm that uses graph theoretic and computational geometry constructs is used to determine the best- and worst-case coverages.

Radar and sonar coverage also present several related challenges. Based on the measured radar cross sections and the coverage diagrams for the different radars, [11] proposes a method for optimally locating the radars to achieve satisfactory surveillance with limited radar resources.

Sensor placement on two- and three-dimensional grids has been formulated as combinatorial optimization and coding theory problems, and solved using integer linear programming [4]. This approach suffers from two main drawbacks. First, computational complexity makes the approach infeasible for large problem instances. Second, the grid coverage approach relies on "perfect" sensor detection, i.e. a sensor is expected to yield a binary yes/no detection outcome in every case. It is well known however that there is inherent uncertainty associated with sensor readings; hence sensor detections must be modeled probabilistically. A probabilistic optimization framework for minimizing the number of sensors for a two-dimensional grid has also been proposed [8]. These algorithms attempt to maximize the average coverage of the grid points. However, [8] assume that there is no uncertainty associated with the predetermined sensor locations. This may not be a feasible assumption in most practical cases, e.g., when sensors are airdropped.

Self-deployment for mobile sensors based on the notion of potential fields is presented in [12]. However, self-deployment does not provide a solution for case of static sensors that need to be deployed in a specific configuration for applications such as environmental monitoring. In [15], the authors propose a heuristic power-efficient sensor deployment strategy by selecting mutually-exclusive sensor sets to cover the sensor field. The sensors in only one set are awake at a time; the others are asleep. An energy-efficient strategy for target localization is proposed in [16]. The concept of virtual force is used to achieve rapid sensor deployment as an improvement over random placement. A related problem in wireless sensor networks is that of spatial localization [7], [9]. A number of techniques for both fine and coarse-grained localization have been proposed [9], [13].

\section{Sensor AND TerRain Model}

Sensor placement requires accurate yet computationally feasible sensor detection models. In this work, we first assume that the sensor field is made up of grid points. The granularity of the grid (distance between consecutive grid points) is determined by the accuracy with which the sensor placement is desired.

Let us consider a sensor field represented by a $m \times n$ grid. We will use Grid to denote the set of grid points in the sensor field. Let $S$ denoted the set of deployed sensors and let the corresponding sensor locations be $L_{s}=\left\{\left(x_{i}, y_{i}\right) \mid s_{i}\right.$ at $\left(x_{i}, y_{i}\right)$, $\left.s_{i} \in S\right\}$. We assume that the probability of detection of a target by a sensor varies exponentially with the distance between the target and the sensor. The probability that a target at grid point $(i, j)$ is detected by a sensor at grid point $(x, y)$, denoted as $c_{i j}(x, y)$, is expressed as:

$$
c_{i j}(x, y)=e^{-\alpha d_{i j}(x, y)}
$$

where $d_{i j}(x, y)$ is the Euclidean distance between $(i, j)$ and $(x, y)$, i.e., $d_{i j}(x, y)=\sqrt{(x-i)^{2}+(y-j)^{2}}$, and $\alpha$ is a parameter representing the physical characteristics of the sensor. This is also the coverage confidence level of this point. The parameter $\alpha$ can be used to model the quality of the sensor and the rate at which its detection probability diminishes with distance. Clearly, the detection probability is 1 if the target location and the sensor location coincide. For any two grid points $(i, j)$ and $(x, y)$ in the sensor field, we associate two probability values: (i) $p_{i j}(x, y)$, which denotes the probability that a target at grid point $(i, j)$ is detected by a sensor at grid point $(x, y)$; (ii) $p_{x y}(i, j)$, which denotes the probability that a target at grid point $(x, y)$ is detected by a sensor at grid point $(i, j)$. In the absence of obstacles, these values are symmetric, i.e. $p_{i j}(x, y)=p_{x y}(i, j)$. As each point on the grid is a possible location for a sensor, there is a coverage probability matrix associated with each grid point $(x, y)$, where a sensor may be placed. This is denoted as $C_{x y}=\left[c_{i j}(x, y)\right]_{m \times n}$, where $m$ and $n$ correspond to the grid dimensions in $x$ and $y$ dimensions respectively. The presence of obstacles can be modeled by altering the detection probabilities for appropriate pairs of grid points. Note that the choice of a sensor detection model does not limit the applicability of the placement algorithm in any way. The detection model is simply an input parameter to the placement algorithm. Alternative detection models can therefore be considered without requiring a major redesign of the placement algorithm.

\section{Modeling of Non-Deterministic Placement}

During sensor deployment, an attempt is made to place sensors at appropriate predetermined locations by by airdropping or other means. This does not guarantee however that sensors are actually placed at the designated positions, due to unanticipated conditions such as wind, the slope of the terrain, etc. In this case, there is a certain probability of a sensor being located at a particular grid point as a function of the designated location. The deviation about the designated 
sensor locations may be modeled using a Gaussian probability distribution, where the intended coordinates $(x, y)$ serve as the mean values with standard deviation $\sigma_{x}$ and $\sigma_{y}$ in the $x$ and $y$ dimensions, respectively. Assuming that the deviations in the $x$ and $y$ dimensions are independent, the joint probability density function with mean $(x, y)$ is given by:

$$
p_{x y}\left(x^{\prime}, y^{\prime}\right)=\frac{e^{-\frac{\left(x^{\prime}-x\right)^{2}}{2 \sigma_{x}^{2}}-\frac{\left(y^{\prime}-y\right)^{2}}{2 \sigma_{y}^{2}}}}{2 \pi \sigma_{x} \sigma_{y}} .
$$

Let us use $A$ to denote the total area encompassing all possible sensor locations. To model the uncertainty in sensor locations, the conditional probability $c_{i j}^{*}(x, y)$ for a grid point $(i, j)$ to be detected by a sensor that is supposed to be deployed at $(x, y)$ is then given by:

$$
c_{i j}^{*}(x, y)=\frac{\sum_{\left(x^{\prime}, y^{\prime}\right) \in A} c_{i j}\left(x^{\prime}, y^{\prime}\right) p_{x y}\left(x^{\prime}, y^{\prime}\right)}{\sum_{\left(x^{\prime}, y^{\prime}\right) \in A} p_{x y}\left(x^{\prime}, y^{\prime}\right)} .
$$

Based on Equations (2) and (3), we defined the matrices $C_{x y}^{*}=$ $\left[c_{i j}(x, y)\right]_{m \times n}$ and $P=\left[p_{x y}\left(x^{\prime}, y^{\prime}\right)\right]_{A}$.

\section{Sensor Placement Algorithms}

The goal of sensor placement algorithms is to determine the minimum number of sensors and their locations such that every grid point is covered with a minimum confidence level. The sensor placement algorithms do not give us the actual location of a sensor but only the mean position of the sensor. It is straightforward to define the miss probability in our sensor deployment scenario. The miss probability of a grid point $(i, j)$ due to a sensor at $(x, y)$, denoted as $m_{i j}(x, y)$, is given by:

$$
m_{i j}(x, y)=1-c_{i j}^{*}(x, y) .
$$

Therefore the miss probability matrix due to a sensor placed at $(x, y)$ is $M_{x y}=\left[m_{i j(x, y)}\right]_{m \times n} . M_{x y}$ is associated with each grid point and can be pre-determined based on Equations (2), (3) and (4). Since a number of sensors are placed for coverage, we would like to know the miss probability of each grid point due to a set of sensors, namely the collective miss probability. We denote the term collective miss probability as $m_{i j}$ and define it in the form of maximum likelihood function as

$$
m_{i j}=\prod_{(x, y) \in L_{s}} m_{i j}(x, y)=\prod_{(x, y) \in L_{s}}\left[1-c_{i j}^{*}(x, y)\right] .
$$

Accordingly we have $M=\left[m_{i} j\right]_{m \times n}$ as the collective miss probability matrix over the grid points in the sensor field.

We determine the location of the sensors one at a time. In each step, we find all possible locations that are available on the grid for a sensor, and calculate the overall miss probability associated due to this sensor and those already deployed. We denote the overall miss probability due to the newly introduced sensor at grid point $(x, y)$ as $\widetilde{m}(x, y)$, which is defined as

$$
\widetilde{m}(x, y)=\sum_{(i, j) \in G r i d} m_{i j}(x, y) m_{i j} .
$$

Based on the $\widetilde{m}(x, y)$ values, where $(x, y) \in$ Grid and $(x, y) \notin L_{s}$, we can place sensors either at the grid point with the maximum miss probability (the worst coverage case) or the minimum miss probability (the best coverage case). We refer to the two strategies as MAX_MISS and MIN_MISS, respectively. Therefore, the sensor location can be found based on the following rule. For $(x, y) \in \operatorname{Grid}$ and $(x, y) \notin L_{s}$,

$$
\widetilde{m}(x, y)= \begin{cases}\min \left\{\widetilde{m}\left(x^{\prime}, y^{\prime}\right)\right\}, & \text { if MIN_MISS is used; } \\ \max \left\{\widetilde{m}\left(x^{\prime}, y^{\prime}\right)\right\}, & \text { if MAX_MISS is used. }\end{cases}
$$

When the best location is found for the current sensor, the collective miss probability matrix $M$ is updated with the newly introduced sensor at location $(x, y)$. This is carried out using Equation (8):

$$
M=M \cdot M_{i j}(x, y)=\left[m_{i j} \cdot m_{i j}(x, y)\right]_{m \times n} .
$$

There are two parameters that serve as the termination criterion for the two algorithm. The first is $k_{\max }$, which is the maximum number of sensors that we can afford to deploy. The second is the threshold on the miss probability of each grid point, $m_{t h}$. Our objective is to ensure that every grid point is covered with probability at least $c_{t h}=1-m_{t h}$. Therefore, the rule to stop the further execution of the algorithm is

$$
m_{i j}<m_{t h} \text { for all }(i, j) \in G \text { rid or } k>k_{\max }
$$

where $k$ is the number of deployed sensors. The performance of the proposed algorithm is evaluated using the average coverage probability of the grid defined as

$$
c_{\text {avg }}=\frac{\sum_{(x, y) \in G r i d} c_{i j}}{m \cdot n} \text {. }
$$

where $c_{i j}$ is the collective coverage probability of a grid point due to all sensors on the grid, defined as

$$
\begin{aligned}
c_{i j} & =1-\prod_{(x, y) \in L_{s}} m_{i j}(x, y) \\
& =1-\left\{\prod_{(x, y) \in L_{s}}\left[1-c_{i j}^{*}(x, y)\right]\right\} .
\end{aligned}
$$

Note that matrices $C_{x y}, M_{x y}$ and $P_{A}$ can all be calculated before the actual execution of the placement algorithms. This is illustrated in Fig. 2 as the pseudocode for the initialization procedure. The initialization procedure is the algorithm overhead which has a complexity of $O\left((m n)^{2}\right)$, where the dimension of the grid is $m \times n$. Once the initialization is done, we may apply either MIN_MISS or MIN_MISS uncertaintyaware sensor placement algorithm using different values for $m_{t h}$ and $k_{\max }$ with the same $C_{x y}, M_{x y}$ and $P_{A}$. Fig. 3 outlines the main part in pseudocode for the uncertainty-aware sensor placement algorithms. The computational complexity for both MIN_MISS and MAX_MISS is $O(m n)$. 


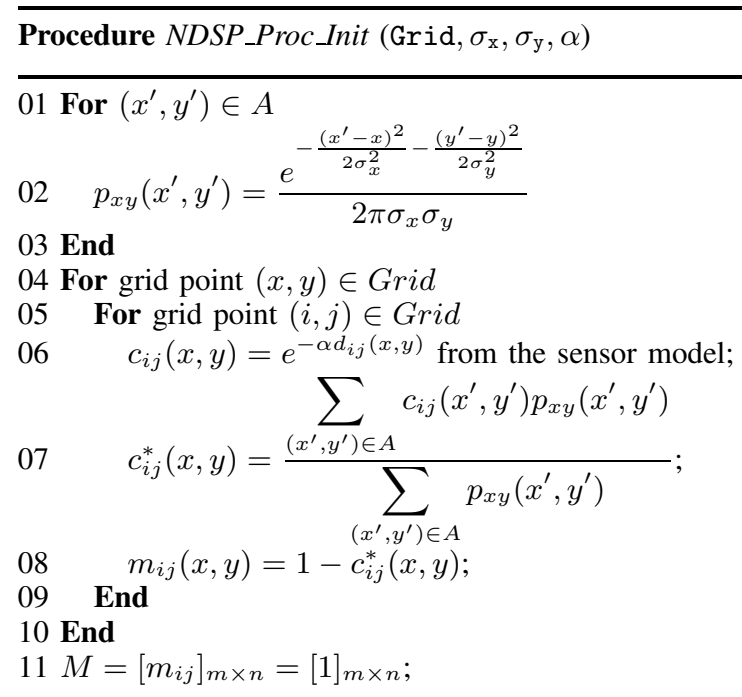

Fig. 2. Initialization pseudocode.

Procedure NDSP_Proc_Main (type, $\left.\mathrm{k}_{\max }, \mathrm{m}_{\mathrm{th}}, \mathrm{Grid}, \mathrm{C}_{\mathrm{xy}}, \mathrm{M}_{\mathrm{xy}}, \mathrm{P}_{\mathrm{A}}, \mathrm{M}\right)$

01 Set $S=\{\phi\} ; L_{s}=\{\phi\} ; k=|S|$;

02 Repeat

03 For grid point $(x, y) \in \operatorname{Grid}$ And $(x, y) \notin L_{s}$

$04 \quad \tilde{m}(x, y)=\sum_{(i, j) \in \text { Grid }} m_{i j}(x, y) m_{i j}$;

05 End

06 If type $=$ MIN_MISS

$07 \quad \widetilde{m}(x, y)=\min \left\{\widetilde{m}\left(x^{\prime}, y^{\prime}\right)\right\},\left(x^{\prime}, y^{\prime}\right) \in$ Grid;

08 Else /* MAX_MISS */

$09 \quad \widetilde{m}(x, y)=\max \left\{\widetilde{m}\left(x^{\prime}, y^{\prime}\right)\right\},\left(x^{\prime}, y^{\prime}\right) \in$ Grid;

10 End

11 Set $k=k+1 ; L_{s}=L_{s} \cup\{(x, y)\} ; S=S \cup\left\{s_{k}\right\}$;

12 For grid point $(i, j) \in$ Grid

$13 \quad m_{i j}=m_{i j} \cdot m_{i j}^{*}(x, y)$;

14 End

15 Until $m_{i j}<m_{t h}$ for all $(i, j) \in$ Grid Or $k>k_{\text {max }}$;

Fig. 3. Pseudocode for sensor placement algorithm.

\section{Simulation Results}

We evaluated the proposed uncertainty-aware sensor placement algorithms MIN_MISS and MAX_MISS through simulations. Note that for practical reasons, we use a truncated Gaussian model because the sensor deviations in a sensor location are unlikely to span the complete sensor field. Therefore $x^{\prime}-x$ and $y^{\prime}-y$ in Equation (2) are limited to a certain range, which reflects how large the variation is in the sensor locations during the deployment. The maximum error in $x$ direction is denoted as $e_{\max }^{x}=\max \left(x^{\prime}-x\right)$, and the maximum error in $y$ direction is denoted as $e_{\max }^{y}=\max \left(y^{\prime}-y\right)$. We then present our simulation results for different sets of parameters in units of grid point where $m=n=10, \alpha=0.5$, $\sigma_{x}=\sigma_{y}=0.1,0.32,1,2$, and $e_{\max }^{x}=e_{\max }^{y}=2,3,5$.

Fig. 4 presents the result for the two sensor placement algorithms described by Equation (7). Fig. 5 compares the

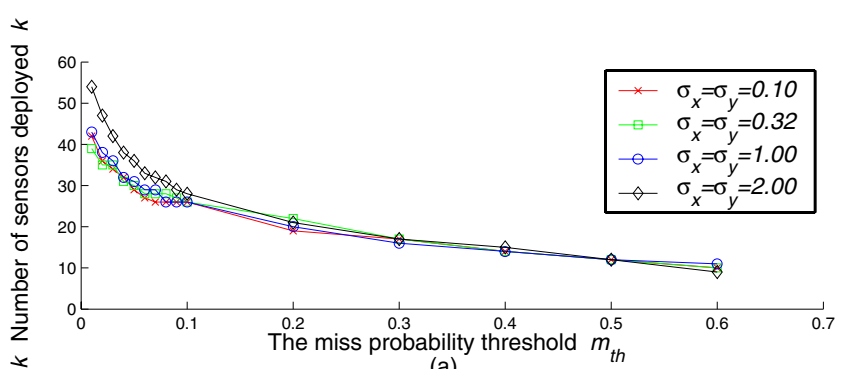

(a)

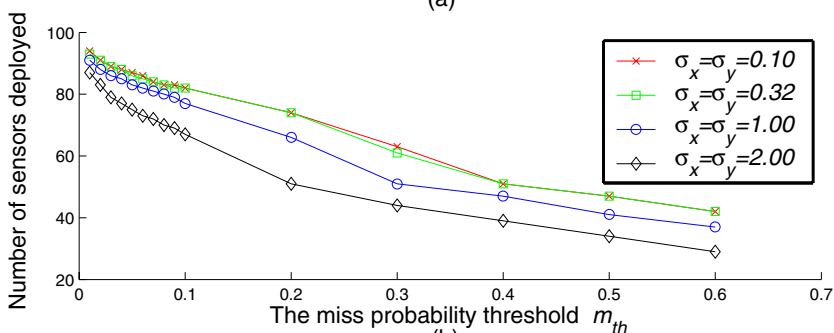

(b)

Fig. 4. Number of sensors required as a function of the miss probability threshold with $\alpha=0.5, e_{\max }^{x}=e_{\max }^{y}=5$, for (a) MIN_MISS (b) MAX_MISS.
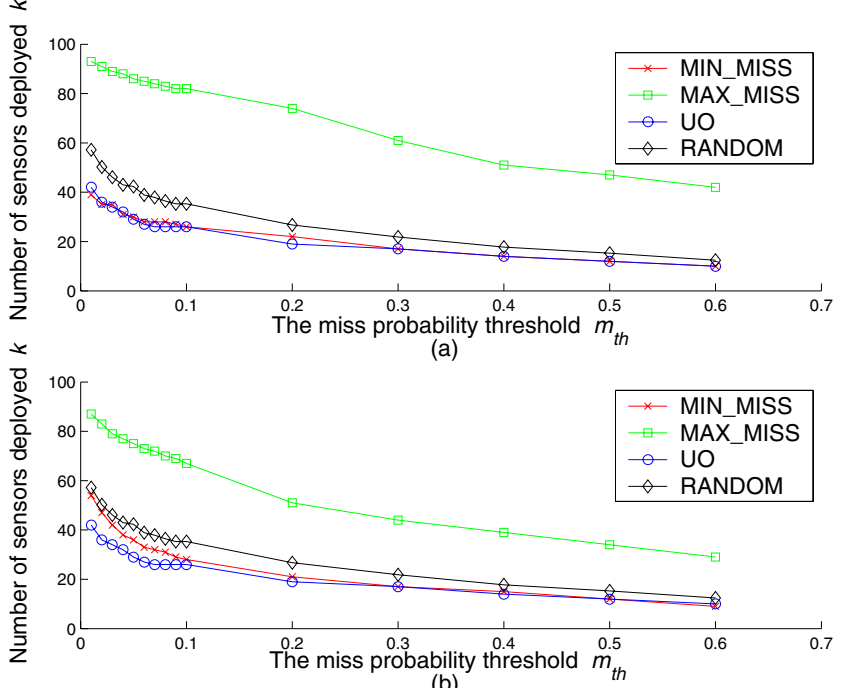

(b)

Fig. 5. Number of sensors required for various placement schemes with $\alpha=0.5, e_{\max }^{x}=e_{\max }^{y}=5$, and (a) $\sigma_{x}=\sigma_{y}=0.32$ (b) $\sigma_{x}=\sigma_{y}=2$.

proposed MIN_MISS and MAX_MISS algorithms with the base case where no location errors are considered, i.e. an uncertainty-oblivious (UO) strategy is followed by setting $\sigma_{x}=\sigma_{y}=0$. We also consider a random deployment of sensors. The results show that MIN_MISS is nearly as efficient as the base uncertainty-oblivious algorithm, yet it is much more robust since it can better tolerate errors than MAX_MISS and random placement algorithms. Fig. 6 presents results for the truncated Gaussian models with different maximum errors. Compared to random deployment, MIN_MISS requires more sensors here but we expect random deployment to perform worse in the presence of obstacles.

Fig. 7 compares the coverage based on Equation (10) for MIN_MISS and MAX_MISS with coverage obtained without location uncertainty. The results show that the MAX_MISS algorithm, which place more sensors for a given coverage 


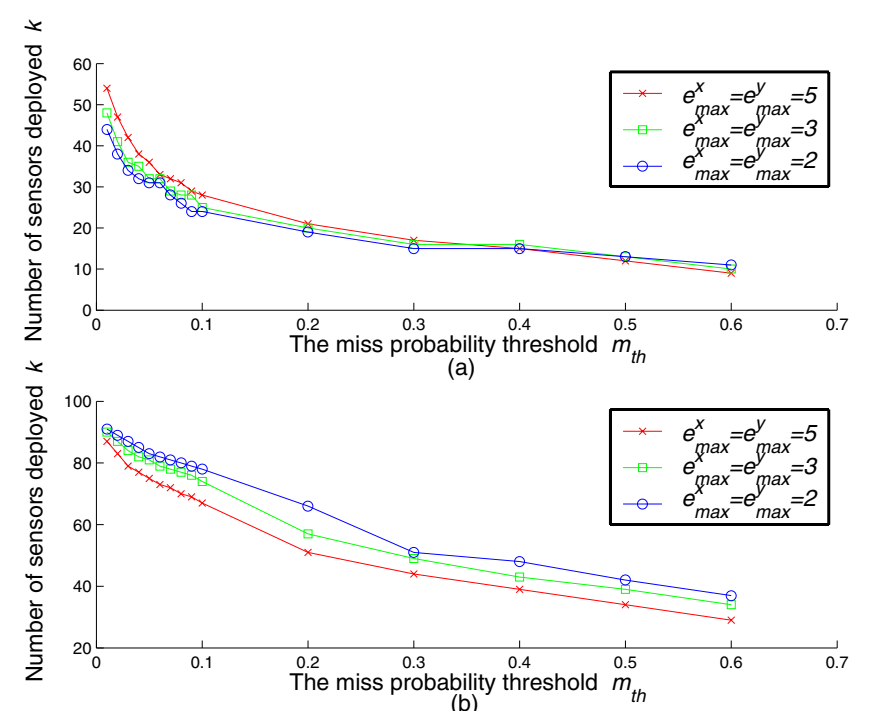

(b)

Fig. 6. Comparisons in different truncated Gaussian models with $\alpha=$ $0.5, \sigma_{x}=\sigma_{y}=2$ for (a) MIN_MISS (b) MAX_MISS.

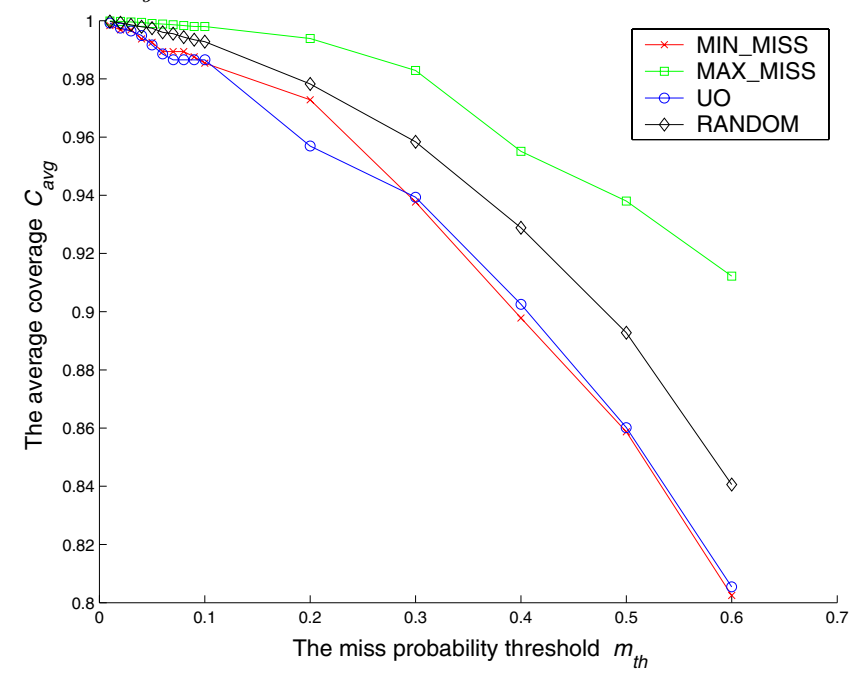

Fig. 7. Comparison in average coverage for various placement schemes with $\alpha=0.5, e_{\max }^{x}=e_{\max }^{y}=5, \sigma_{x}=\sigma_{y}=0.32$.

threshold, provides higher overall coverage.

\section{CONCLUSIONS}

We have formulated an optimization problem on sensor placement in the context of uncertainty-aware sensor deployment, wherein a minimum number of sensors are deployed to provide sufficient grid coverage of the sensor field. The sensor location has been modeled as a random variable with a Gaussian probability distribution. We have presented two polynomial-time algorithms to optimize the number of sensors and determine their placement in an uncertainty-aware manner.

We are currently extending this work to the case of correlated sensor detections, and sensors where the observable domain is defined by minimum and maximum range, and an angular width. Also, we are investigating mobile sensors and obstacles. We are examining how in addition to determining a sensor location in each iteration, our algorithm can also determine an appropriate sensor from a set of candidate sensors of the same modality. While the proposed framework does not depend on any particular sensor or terrain model, we are extending this work to real environments where various types of uncertainty distributions may apply. Nevertheless, this work is expected to eventually pave the way for an integrated framework for sensor placement that incorporates power management and fault tolerance.

\section{ACKNOWLEDGEMENT}

This research was supported in part by ONR under grant no. N66001-00-1-8946. It was also sponsored in part by DARPA, and administered by the Army Research Office under Emergent Surveillance Plexus MURI Award No. DAAD19-01-1-0504. Any opinions, findings, and conclusions or recommendations expressed in this publication are those of the authors and do not necessarily reflect the views of the sponsoring agencies.

\section{REFERENCES}

[1] G. J. Pottie and W. J. Kaiser, "Wireless sensor networks", Communications of the ACM, vol. 43, pp. 51-58, May 2000.

[2] T. Clouqueur, V. Phipatanasuphorn, P. Ramanathan and K. K. Saluja, "Sensor Deployment for Target Detection", Proc. International Workshop on Wireless Sensor Networks and Applications, pp. 42-48, Sep. 2002.

[3] N. S. V. Rao, S. S. Iyengar, B. J. Oomen and R. L. Kashyap, "On terrain model acquisition by a point robot amidst polyhedral obstacles", IEEE Journal of Robotics and Automation, vol. 4, pp. 450-455, August 1988.

[4] K. Chakrabarty, S. S. Iyengar, H. Qi and E. Cho, "Grid coverage for surveillance and target location in distributed sensor networks", IEEE Transactions on Computers, vol. 51, pp. 1448-1453, December 2002.

[5] S. Meguerdichian, F. Koushanfar, M. Potkonjak and M. B. Srivastava, "Coverage problems in wireless ad-hoc sensor networks", Proc. IEEE Infocom Conference, vol 3, pp. 1380-1387, 2001.

[6] S. Meguerdichian, F. Koushanfar, G. Qu and M. Potkonjak, "Exposure in wireless ad-hoc sensor networks", Proc. Mobicom Conference, pp. 139150 , July 2001

[7] N. B. Priyantha, A. Chakraborty and H. Balakrishnan, "The cricket location-support system", Proc. ACM/IEEE International Conference on Mobile Computing and Networking, 2000.

[8] S. S. Dhillon and K. Chakrabarty, "Sensor placement for effective coverage and surveillance in distributed sensor networks", Proc. IEEE Wireless Communications and Networking Conference, pp. 1609-1614, 2003.

[9] N. Bulusu, J. Heidemann and D. Estrin, "GPS-less low-cost outdoor localization for very small devices", IEEE Personal Communication Magazine, vol. 7, no. 5, pp. 28-34, Oct 2000.

[10] S. A. Musman, P.E. Lehner and C. Elsaesser, "Sensor planning for elusive targets", Journal of Computer and Mathematical Modeling, vol. 25, No. 3, pp. 103-115, 1997.

[11] T. Kasetkasem and P. K. Varshney, "Communication Structure Planning for Multisensor Detection Systems", Proc. IEE Conference on Radar, Sonar and Navigation, vol. 148, pp. 2-8, Feb. 2001.

[12] A. Howard, M. J. Matarić, and G. S. Sukhatme, "Mobile sensor network deployment using potential fields: A distributed, scalable solution to the area coverage problem", Proc. International Symposium on Distributed Autonomous Robotics Systems, pp. 299-308, 2002.

[13] N. Bulusu, J. Heidemann and D. Estrin, "Adaptive beacon placement", Proc. International Conference on Distributed Computing Systems, pp. 489-498, 2001.

[14] J. Heidemann and N. Bulusu, "Using geospatial information in sensor networks", Proc. CSTB workshop on Intersection of Geospatial Information and Information Technology, October 2001.

[15] S. Slijepcevic and M. Potkonjak, "Power Efficient Organization of Wireless Sensor Networks", Proc. IEEE International Conference on Communications, pp. 472-476, 2001.

[16] Y. Zou and K. Chakrabarty, "Sensor deployment and target localization based on virtual forces", Proc. IEEE Infocom Conference, pp. 1293-1303, 2003. 